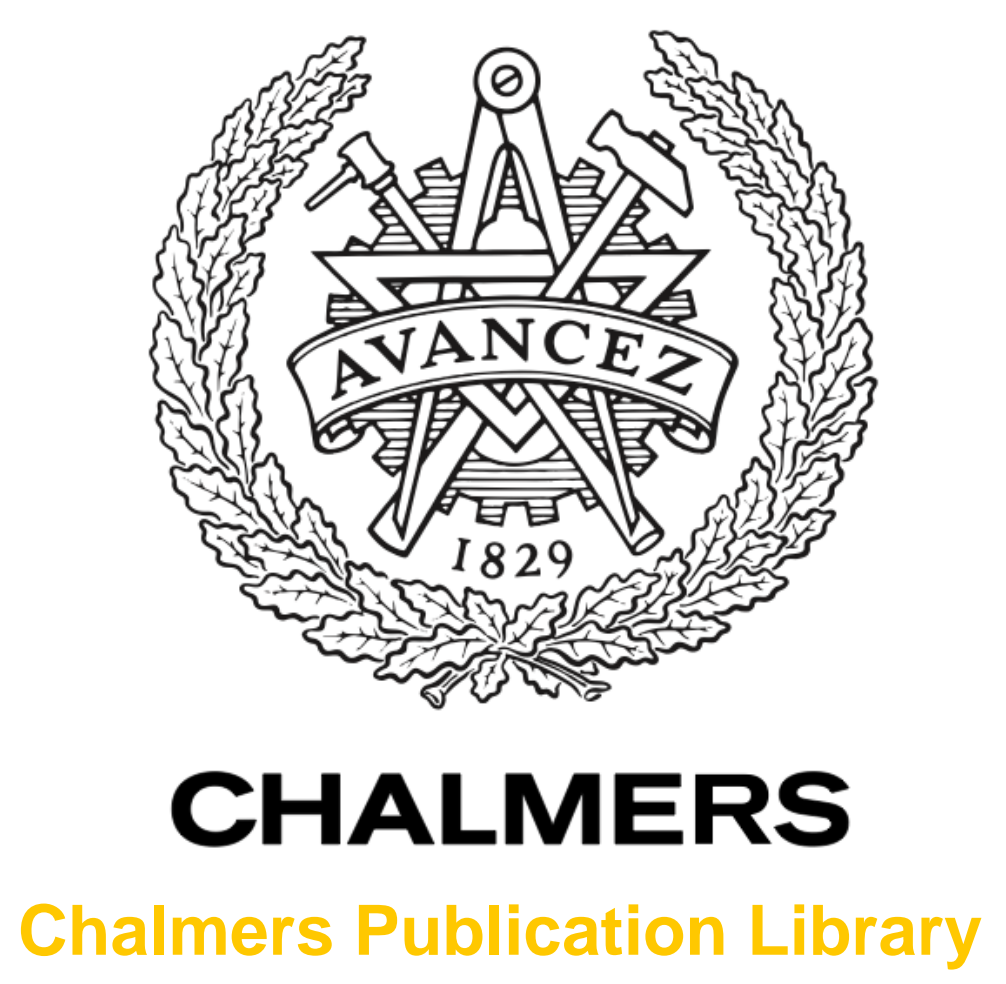

\author{
Simulations of a planar array arrangement for automotive Random-LOS OTA \\ testing
}

This document has been downloaded from Chalmers Publication Library (CPL). It is the author's version of a work that was accepted for publication in:

2016 10th European Conference on Antennas and Propagation, EuCAP 2016

\begin{abstract}
Citation for the published paper:
Glazunov, A. ; Razavi, A. ; Kildal, P. (2016) "Simulations of a planar array arrangement for automotive Random-LOS OTA testing". 2016 10th European Conference on Antennas and Propagation, EuCAP 2016 pp. 7481285.
\end{abstract}

http://dx.doi.org/10.1109/EuCAP.2016.7481285

Downloaded from: http://publications.lib.chalmers.se/publication/246540

Notice: Changes introduced as a result of publishing processes such as copy-editing and formatting may not be reflected in this document. For a definitive version of this work, please refer to the published source. Please note that access to the published version might require a subscription.

Chalmers Publication Library (CPL) offers the possibility of retrieving research publications produced at Chalmers University of Technology. It covers all types of publications: articles, dissertations, licentiate theses, masters theses, conference papers, reports etc. Since 2006 it is the official tool for Chalmers official publication statistics. To ensure that Chalmers research results are disseminated as widely as possible, an Open Access Policy has been adopted.

The CPL service is administrated and maintained by Chalmers Library. 


\title{
Simulations of a Planar Array Arrangement for Automotive Random-LOS OTA Testing
}

\author{
Andrés Alayón Glazunov, Aidin Razavi, and Per-Simon Kildal \\ Department of Signals and Systems, Chalmers University of Technology, Gothenburg, Sweden
}

\begin{abstract}
We present simulations of near-field plane wave synthesis by a planar array. The focus is on minimization of reference signal errors within the test zone for Random Line-OfSight Over-The-Air characterization of wireless devices on cars. The analysis considers the output of the ideal digital threshold receiver model of the device under test as a Probability of Detection curve. The dimensions, the interelement spacings and the number of elements in a planar array comprising Huygens sources are investigated to produce an absolute error less than $0.5 \mathrm{~dB}$.
\end{abstract}

\section{INTRODUCTION}

Driverless cars may provide a number of advantages compared to current non-driverless cars, e.g., reduced traffic congestion, increased roadway capacity and improved environmental footprint. Over-The-Air (OTA) characterization of wireless devices on car is essential to help building communication systems so that different vehicles can talk to each other and surrounding equipment in a reliable way.

OTA testing is mainly concerned with measuring the performance of the whole device including the antennas [1]. Compact reverberation chambers (with reflecting walls) and anechoic chambers (with absorbing walls) have the potential to be used for OTA characterization of wireless devices on cars [2]. On one hand, reverberation chambers have longer been known to be useful for both passive and active OTA testing in RIMP (Rich Isotropic MultiPath) channels [1], [3]. On the other hand, the new compact anechoic (or even semi-anechoic) chamber is used to emulate the so-called Random Line-ofSight (RLOS) propagation channel. The RLOS channel model assumes that the LOS wave will be (in the limiting case) the only contribution to the receive signal at the car, e.g., in highways or in rural areas. The LOS wave can then be treated as a random variable in azimuth due to the arbitrary orientation of the vehicles relative the base stations [4]. For handset tests the polarization in LOS may be considered random too [5].

The present work follows the real-life hypothesis in [4]: if a wireless device is proven to work well in RIMP and RLOS, it will work well in all real-life environments. Here we focus on the performance characterization in the RLOS propagation channel. We make use of the ideal digital threshold receiver model and the concept of probability of detection (PoD) of communication bitstreams [6]. In spite of its simplicity, this model provides an accurate prediction of the OTA throughput of LTE (Long Term Evolution) devices [7]. For example, the STD (Standard Deviation) of the error between measurements and simulations is of the order of $0.2 \mathrm{~dB}$ for efficiency- and power-related quantities in RIMP [7], [8].

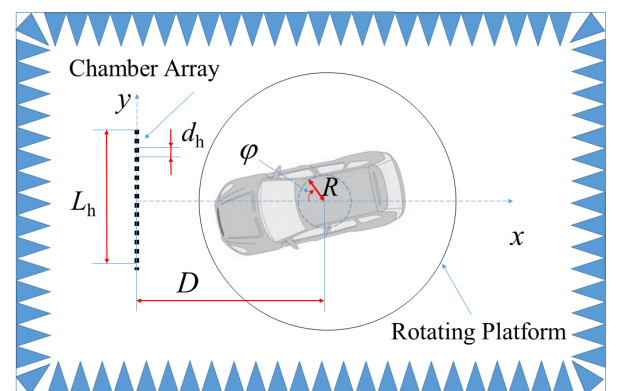

Fig. 1. RLOS OTA geometry in anechoic chamber. The planar chamber array has horizontal dimension $L_{\mathrm{h}}$ and vertical dimension $L_{\mathrm{v}}$ (not shown).

The first experimental results for the OTA characterization of an active LTE MIMO (shark fin) antenna on car in RLOS are given in [9]. Following the real-life hypothesis stated above, a first experimental comparison of the OTA characterization of this antenna in both RIMP and RLOS can be found in [10]. In both these studies we used a single bowtie element antenna to simulate a base station antenna (the chamber antenna) to communicate with the DUT (Device Under Test) on the car. In spite of the encouraging results, a much larger antenna must be used. This is needed to obtain a large enough test zone emulating an incident plane wave at the DUT. A preliminary study of the reference signal variations within a horizontal circle obtained using a horizontal array was presented in [11]. There we showed that a variation of the order of STD $\sim 0.7 \mathrm{~dB}$ within a circle of radius $R=1 \mathrm{~m}$ can be obtained with a $3 \mathrm{~m}$ long horizontal uniform linear array of vertically polarized Huygens sources. In this paper we look further to control signal variations of a similar order of magnitude, or less, over a horizontal circular area. To achieve this we look into the problem of plane wave synthesis with a uniform planar array [12], [13].

\section{RLOS OTA SETUP}

We focus on practical MIMO algorithms for 1-bitstream and for 2-bitstreams specified in the LTE/LTE-A standards and for future $5 \mathrm{G}$ systems. These are the Maximum Ratio Combining (MRC) and the zero-forcing (ZF) receiver algorithms for single-user implementations [7], [14]. Hence, the OTA characterization system must ensure that two orthogonal polarizations (one vertical and one horizontal or slant $\pm 45^{\circ}$ ) are produced to be able to test MIMO in a RLOS channel.

The cost-efficiency requirements push us towards RLOS OTA solutions integrated with a small anechoic chamber 


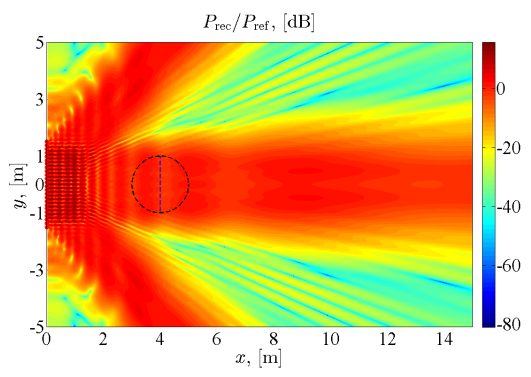

(a)

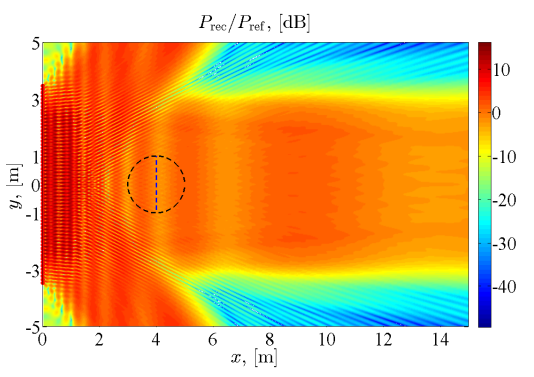

(b)

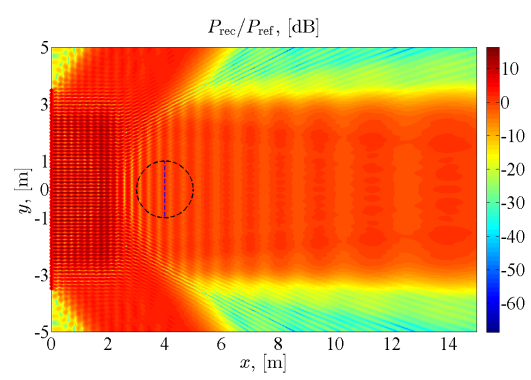

(c)

Fig. 2. Normalized received power from planar array of Huygens source elements. Amplitude taper in horizontal direction only. The test zone is given by the circle. (a) $L_{\mathrm{h}}=L_{\mathrm{v}}=3.08 \mathrm{~m}, d_{\mathrm{h}}=d_{\mathrm{h}}=0.1309 \mathrm{~m}, N_{\mathrm{h}}=N_{\mathrm{v}}=24$, (b) $L_{\mathrm{h}}=3.08 \mathrm{~m}, d_{\mathrm{h}}=0.1309 \mathrm{~m}, N_{\mathrm{h}}=58$, (c) $L_{\mathrm{h}}=L_{\mathrm{v}}=7.00 \mathrm{~m}$, $d_{\mathrm{h}}=d_{\mathrm{h}}=0.1216 \mathrm{~m}, N_{\mathrm{h}}=N_{\mathrm{v}}=58$. See Fig.1 for geometry.

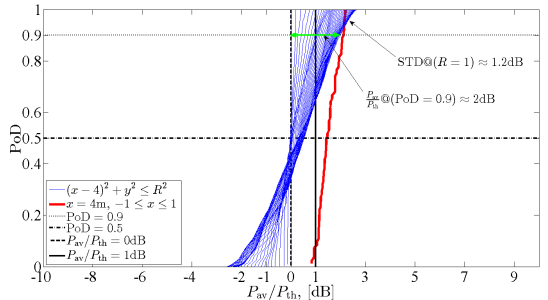

(a)

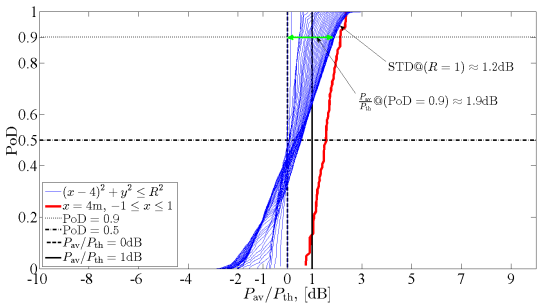

(b)



(c)

Fig. 3. PoD corresponding to different radius of the test zone in Fig.2. Amplitude taper in horizontal direction only. The test zone is given by the circle. (a) $L_{\mathrm{h}}=L_{\mathrm{v}}=3.08 \mathrm{~m}, d_{\mathrm{h}}=d_{\mathrm{h}}=0.1309 \mathrm{~m}, N_{\mathrm{h}}=N_{\mathrm{v}}=24$, (b) $L_{\mathrm{h}}=3.08 \mathrm{~m}, d_{\mathrm{h}}=0.1309 \mathrm{~m}, N_{\mathrm{h}}=58$, (c) $L_{\mathrm{h}}=L_{\mathrm{v}}=7.00 \mathrm{~m}, d_{\mathrm{h}}=d_{\mathrm{h}}=0.1216 \mathrm{~m}$, $N_{\mathrm{h}}=N_{\mathrm{v}}=58$. See Fig.1 for geometry.

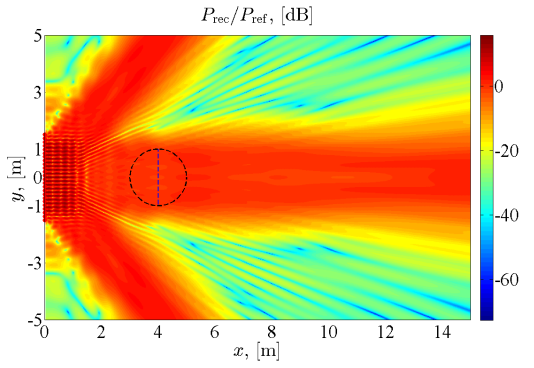

(a)

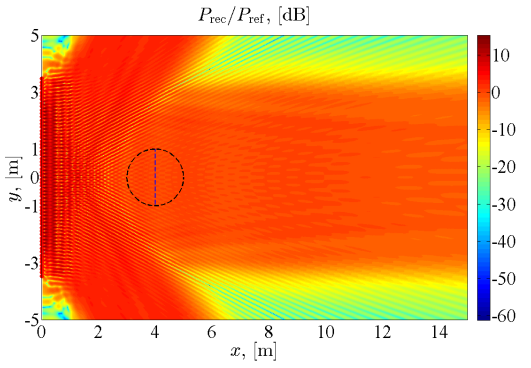

(b)

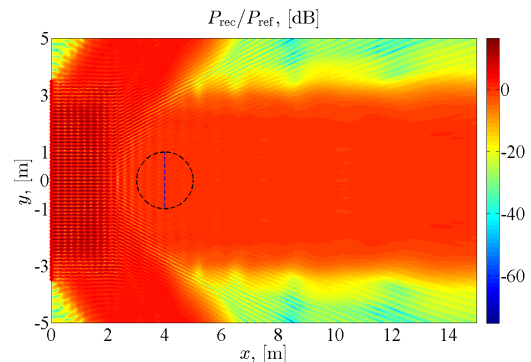

(c)

Fig. 4. Normalized received power from planar array of Huygens source elements. Amplitude taper in both horizontal and vertical directions. The test zone is given by the circle. (a) $L_{\mathrm{h}}=L_{\mathrm{v}}=3.08 \mathrm{~m}, d_{\mathrm{h}}=d_{\mathrm{h}}=0.1309 \mathrm{~m}, N_{\mathrm{h}}=N_{\mathrm{v}}=24$, (b) $L_{\mathrm{h}}=3.08 \mathrm{~m}, d_{\mathrm{h}}=0.1309 \mathrm{~m}, N_{\mathrm{h}}=58,(\mathrm{c}) L_{\mathrm{h}}=L_{\mathrm{v}}=7.00 \mathrm{~m}$, $d_{\mathrm{h}}=d_{\mathrm{h}}=0.1216 \mathrm{~m}, N_{\mathrm{h}}=N_{\mathrm{v}}=58$. See Fig.1 for geometry.

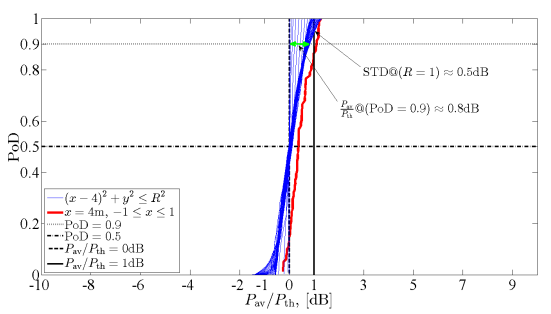

(a)

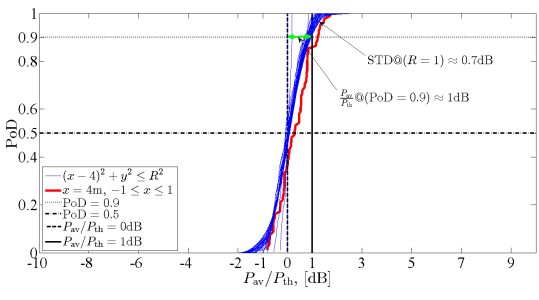

(b)

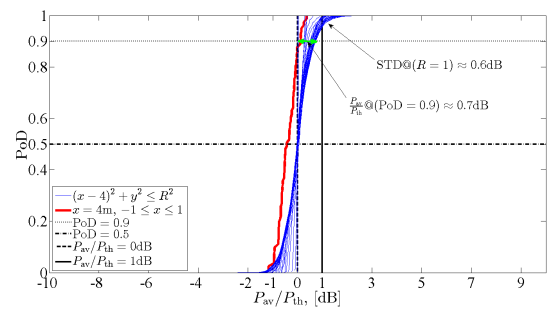

(c)

Fig. 5. PoD corresponding to different radius of the test zone in Fig.4. Amplitude taper in both the horizontal and vertical directions. The test zone is given by the circle. (a) $L_{\mathrm{h}}=L_{\mathrm{v}}=3.08 \mathrm{~m}, d_{\mathrm{h}}=d_{\mathrm{h}}=0.1309 \mathrm{~m}, N_{\mathrm{h}}=N_{\mathrm{v}}=24$, (b) $L_{\mathrm{h}}=3.08 \mathrm{~m}, d_{\mathrm{h}}=0.1309 \mathrm{~m}, N_{\mathrm{h}}=58$, (c) $L_{\mathrm{h}}=L_{\mathrm{v}}=7.00 \mathrm{~m}$, $d_{\mathrm{h}}=d_{\mathrm{h}}=0.1216 \mathrm{~m}, N_{\mathrm{h}}=N_{\mathrm{v}}=58$. See Fig. 1 for geometry.

as shown in Fig.1. In the setup, the car is located on a horizontal turntable at a distance from the chamber antenna. The rotation of the turntable provides the radiating near-field on a cylindrical volume around the vehicle. To achieve the same plane wave front in the near-field as in the far-field of the tested antenna, we use a planar array antenna (i.e., the chamber antenna or chamber array in the following). This chamber array, has a branched beamforming network to obtain the emulated plane wave field directly at the output port of the chamber array [15]. Hence, active wireless communications, as if it was taking place in the far-field of the car or the base station, is practically obtained connecting the output port of the chamber array directly to the wireless communication test instrument. Small elevation angles in the far-field emulating 
base station tilting can be obtained by mechanically tilting the chamber array a few degrees.

A uniform planar array can be considered as an array of linear arrays. In [11] we therefore focused on the horizontal (azimuthal) behaviour of the fields in the test zone due to a horizontal uniform linear array. The test zone is defined by the position of the DUT on the tested car and relative to the axis of the rotating platform shown Fig.1. To simplify the analysis of the chamber array, we assume equidistantly spaced elements. Their positions on the plane of the array are given by the radius vector $\boldsymbol{r}_{n m}=\left(n-\frac{N_{\mathrm{h}}+1}{2}\right) d_{\mathrm{h}} \hat{\boldsymbol{y}}+\left(m-\frac{N_{\mathrm{v}}+1}{2}\right) d_{\mathrm{v}} \hat{\boldsymbol{z}}$ for $n=1,2, \ldots, N_{\mathrm{h}}$ and $m=1,2, \ldots, N_{\mathrm{v}} . \hat{\boldsymbol{y}}$ and $\hat{\boldsymbol{z}}$ are unit vectors pointing along the horizontal and the vertical dimensions of the array, respectively. $d_{\mathrm{h}}$ and $d_{\mathrm{v}}$ are the interelement spacings along the two dimensions of the planar array. We choose to define the total length of the array in one dimension by extending it with half of the interelement spacing outside the first and the last elements. Hence, $L_{\mathrm{h}}=N_{\mathrm{h}} d_{\mathrm{h}}$ and $L_{\mathrm{v}}=N_{\mathrm{v}} d_{\mathrm{v}}$ are the chamber array dimensions in the horizontal and the vertical directions, respectively. The total field at a point $r$ can be computed by superposition

$$
\boldsymbol{E}(\boldsymbol{r})=\sum_{n=1}^{N_{\mathrm{h}}} \sum_{m=1}^{N_{\mathrm{v}}} \boldsymbol{G}\left(\hat{\boldsymbol{r}}_{n m}\right) \frac{e^{-j k\left|\boldsymbol{r}-\boldsymbol{r}_{n m}\right|}}{\left|\boldsymbol{r}-\boldsymbol{r}_{n m}\right|},
$$

where $\boldsymbol{G}\left(\hat{\boldsymbol{r}}_{n m}\right)$ is the far-field function of the element with indices $(n, m)$ when referred to the phase reference point $\boldsymbol{r}_{n m}$ of that element, and $\hat{\boldsymbol{r}}_{n m}=\left(\boldsymbol{r}-\boldsymbol{r}_{n m}\right) /\left|\boldsymbol{r}-\boldsymbol{r}_{n m}\right|$. Let's consider a linearly polarized ideal isotropic antenna oriented along the vertical $z$-axis and located at the test zone. Thus, for this specific case, the normalized received power at point $r$ can be computed as

$$
\frac{P_{\text {rec }}}{P_{\text {ref }}}=\frac{\pi R^{2}\left|E_{\theta}(\boldsymbol{r})\right|^{2}}{\int_{0}^{R} \int_{0}^{2 \pi}\left|E_{\theta}(\boldsymbol{r}(\rho, \varphi))\right|^{2} \rho \mathrm{d} \rho \mathrm{d} \varphi},
$$

where $E_{\theta}=\boldsymbol{E} \cdot \hat{\boldsymbol{\theta}}$, where $\hat{\boldsymbol{\theta}}$ is the unit vector along the $\theta$ axis at the observation point within the circle of radius $R$. It should be noted that $\hat{\boldsymbol{\theta}}=\hat{\boldsymbol{z}}$ in the specific case analyzed in this paper. The distance from the center of the chamber array to the rotating platform axis is given by $D$ as shown in Fig.1. The test zone in RLOS OTA is defined here by the circle of radius $R$ with center at the axis of the rotating platform. The radius $R$ is defined by the positions of a DUT on the lateral sides of the car. Hence, $R$ is approximately equal to half the width of the car.

\section{Probability of Detection}

According to the ideal digital threshold receiver model the error rate goes sharply from only errors to free-of-errors in the Additive White Gaussian Noise (AWGN) channel. The jump occurs at the receiver threshold level and it is system specific. If the channel undergoes fading, then averaging over all the possible fading states gives the normalized average throughput [6]

$$
\frac{\operatorname{TPUT}_{\mathrm{av}}\left(\gamma_{\mathrm{av}} / \gamma_{\mathrm{th}}\right)}{\operatorname{TPUT}_{\max }}=\operatorname{PoD}\left(\gamma_{\mathrm{av}} / \gamma_{\mathrm{th}}\right)=1-\operatorname{CDF}\left(\gamma_{\mathrm{th}} / \gamma_{\mathrm{av}}\right)
$$

where $\mathrm{PoD}$ is the Probability of Detection (PoD) function, CDF is Cumulative Distribution Function (CDF) of the power at the output of the threshold receiver, $\gamma_{\text {av }}$ is the available average received signal-to-noise ratio (SNR) under the AWGN assumption, $\gamma_{\text {th }}$ is the receiver threshold level that may be obtained, e.g., from conducted measurements or other proper OTA reference calibration measurements. The relative throughput is then given by the PoD in (4), which simplifies the throughput performance analysis of wireless devices [16], [17].

\section{Plane WAVE Synthesis AND Simulation RESUlts}

The ideal incident field for RLOS OTA characterization is a uniform plane wave within the test area. In principle, there is no fundamental limitations to generating a perfect plane wave in the near field of sources [12], [13]. However, limitations arise in practical situations from the use of an array of realistic antennas used to approximate the smooth electric current over the aperture of the source. Indeed, a major contribution to the error field within the test zone originates from the edges of the array. This is a direct result of the sudden truncation of the current at the edge of the source region. An undesirable edge diffraction field component interfering with the plane wave field component will cause ripples in the desired region. As shown in [12] the amplitude of the edge diffracted field can be efficiently decreased by tapering the source amplitude at the edge of the array. For a fixed antenna length, the taper causes the width of the test zone to narrow down as the number of tapered elements is increased.

The near-field of the array is comprised by a plane wave field region in the center, two transition diffraction zones each along their respective geometric optics (GO) boundaries, and a very small diffracted field zone outside the transition zone. Hence, in order for the test zone to be within the planewave region the length of the array must satisfy the following condition

$$
L_{\mathrm{h}, \mathrm{v}}>2 R+3 \sqrt{\lambda D / \pi}
$$

where we have assumed that the test zone must be outside the first peak in the transition zone occurring within the $1.5 \sqrt{\lambda D / \pi}$ extents on both sides of the GO boundary [18]. This is evident from [19] and [20].

The planar array in Fig.1 is a broadside array, and the elements are assumed to have uniform phase. Thus, the interelement spacing $d_{\mathrm{h}}$ (or $d_{\mathrm{v}}$ ) can be as large as the wavelength before grating lobes appear. In practice the interelement spacing can be increased to the following value before the grating lobe hits the testing area

$$
d_{\mathrm{h}, \mathrm{v}}<\lambda \sqrt{1+\left(\frac{2 D}{L_{\mathrm{h}, \mathrm{v}}+2 R}\right)^{2}} .
$$

In the above equation, no regard has been taken to the width of the transition region.

Conditions (4) and (5) together with an amplitude taper of the excitations of the antenna elements limit the configuration of the chamber array. In [12], [13] various solutions are 


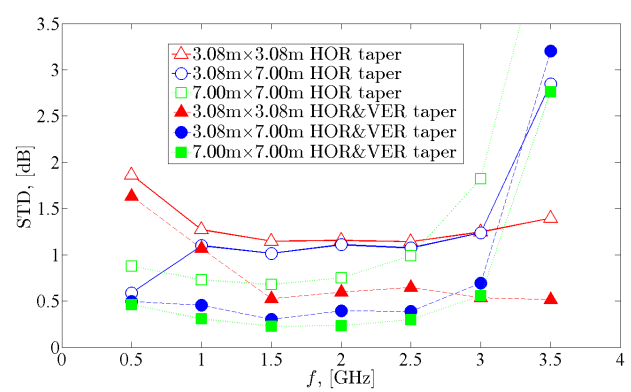

(a)

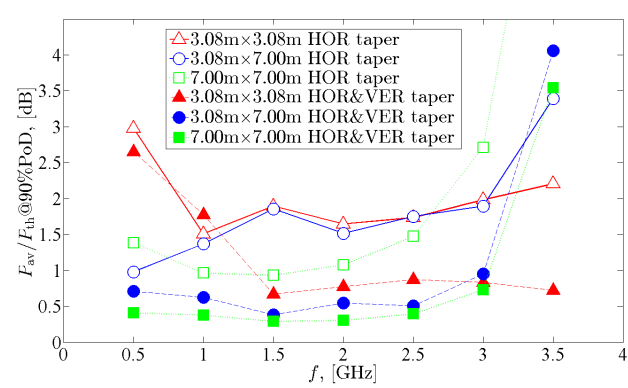

(b)

Fig. 6. (a) Standard deviation and, (b) The difference in $\mathrm{dB}$ between the measured power corresponding to PoD $=0.9$.

proposed to find the optimal excitation (taper) weights that minimize the amplitude and phase ripples in the test zone. In [21] it has been proposed to use a genetic algorithms to solve a global optimization method to find optimal taper weights.

In this contribution we follow the procedure presented in [11]. We start with a horizontal uniform linear array with interelement spacing $d=0.7 \lambda$ to avoid grating lobes to begin with, i.e., since $d<\lambda$. All the elements are assumed to be equal. The Huygens source element has been chosen due to its broad pattern in the forward direction and a null in the backward direction. Our frequency band of interest spans $1.5-3 \mathrm{GHz}$. Therefore we chose the largest frequency within the bandwidth, $f=3 \mathrm{GHz}$, to optimize our array for the plane wave generation in the near-field. The corresponding wavelength is $\lambda=0.1 \mathrm{~m}$. The distance between the chamber array and the axis of the turntable has been chosen as $D=4 \mathrm{~m}$. The goal is to find $L_{\mathrm{h}}, L_{\mathrm{v}}, d_{\mathrm{h}}, d_{\mathrm{v}}, N_{\mathrm{h}}, N_{\mathrm{v}}$ such that a plane wave is generated with the target accuracy within a circle of radius $R=1 \mathrm{~m}$.

The target accuracy is based on the definition of two figures of merit:

1) The difference in $\mathrm{dB}$ between the measured power and the threshold level corresponding to $\mathrm{PoD}=0.9$.

$$
\left(P_{\mathrm{av}} / P_{\mathrm{th}}\right) @ 0.9 \mathrm{PoD} \leq 1 \mathrm{~dB}
$$

where $\mathrm{PoD}^{-1}$ denotes the inverse function operation.

2) The standard deviation of $P_{\mathrm{av}} / P_{\mathrm{th}}$ in $\mathrm{dB}$ scale such that

$$
\mathrm{STD} \leq 1 \mathrm{~dB} .
$$

The PoD is obtained from the measurements of (2) at points within the circle of radius $R$. Hence, the closer the PoDcurve of the reference measurements taken over the area of the test zone to the step function, the better the accuracy of the reference measurements.

Following the procedure in [11] we found that the shortest and thinnest horizontal array satisfying the target accuracy given by (6) and (7) at all radii $0 \leq R \leq 1$ has length $L_{\mathrm{h}}=3.08 \mathrm{~m}$, and $N_{\mathrm{h}}=24$ equidistant Huygens source elements with interelement distance $d_{\mathrm{h}}=1.309 \lambda$. The array length is computed as $L_{\mathrm{h}}=\left(N_{\mathrm{h}}-1\right) d_{\mathrm{h}}+0.07$. For the sake of comparison we also present an optimized array of length $L_{\mathrm{h}}=7.00 \mathrm{~m}$ with $N_{\mathrm{h}}=58$ and $d_{\mathrm{h}}=1.216 \lambda$. In both cases the optimization was performed at $f=3 \mathrm{GHz}$, i.e., $\lambda=0.1 \mathrm{~m}$.
In order to further reduce power variations in the test zone, we also apply a linear-in- $\mathrm{dB}$ amplitude taper from 0 to -6 $\mathrm{dB}$ to $25 \%$ of the elements on each side of the array along one or both array dimensions.

Fig.2 to Fig.5 show simulations results corresponding to linear array size combinations of the considered planar arrays at $f=3 \mathrm{GHz}$, with taper in the horizontal dimension only or with taper in both dimensions. The normalized received powers on the $x-y$ plane for the case when taper is applied only in the horizontal dimension are shown for $L_{\mathrm{h}}=L_{\mathrm{v}}=3.08 \mathrm{~m}$, $L_{\mathrm{h}}=7 \mathrm{~m} \& L_{\mathrm{v}}=3.08 \mathrm{~m}$ and $L_{\mathrm{h}}=L_{\mathrm{v}}=7.00 \mathrm{~m}$, in Fig.2a, Fig.2b and Fig.2c, respectively. Similar plots for the case when taper is applied along both dimensions are shown in Fig.4a, Fig.4b and Fig.4c. By comparing corresponding figures we can see that introducing taper along both dimensions will mitigate the contribution of the diffracted field component coming from the horizontal edges of the planar array. This will result in smaller ripples or signal variations. This can be better observed by looking into the corresponding PoDcurves for the case when taper is used only in the horizontal dimension or in both dimensions as shown in Fig.3 and Fig.5, respectively. The PoD-curves represented by the thin blue lines correspond to circles of various radii from $0.1 \mathrm{~m}$ to $1 \mathrm{~m}$. The PoD-curve represented by the thick red line correspond to the diameter line going in parallel to the planar array; see, e.g., Fig.2a. Both figures of merit according to (6) and (7) are shown for the PoD-curve corresponding to the circle with radius $R=1 \mathrm{~m}$ at each of the subfigures in Fig.3 and Fig.5. One can clearly see that the signal variation along the line parallel to the array is fairly small with a dynamic range of around $1 \mathrm{~dB}$. The position of the corresponding PoD-curve (thick red line) is not centered around the $0 \mathrm{~dB}$ power level since the normalization is taken for variation over the whole circle and not along variation along the line. This also indicates that the signals will vary strongly in other planes parallel to the planar array. The smaller the variation and the more closer the middle of the PoD-curve to the $0 \mathrm{~dB}$ the more closer the synthesized wave is to a plane wave.

Fig.6a and Fig.6b show the standard deviation and the relative power deviation from the ideal reference at $\mathrm{PoD}=0.9$ as function of frequency, respectively. The performance within the target frequency band $1.5-3 \mathrm{GHz}$ is satisfactory and both amplitude taper and increasing the size of the horizontal array 
decrease signal variation. However, performance degradation is observed at lower frequencies, i.e., $f<1.5 \mathrm{GHz}$, for $L_{\mathrm{h}}=L_{\mathrm{v}}=3.08 \mathrm{~m}$. On the other hand, performance degradation is observed at higher frequencies, i.e., $f>3 \mathrm{GHz}$, for the larger arrays $L_{\mathrm{h}}=7.00 \mathrm{~m} \& L_{\mathrm{v}}=3.08 \mathrm{~m}$ and $L_{\mathrm{h}}=$ $L_{\mathrm{v}}=7.00 \mathrm{~m}$. The larger inaccuracy for the smaller array at lower frequencies can be explained by the transition region theory [18] and (4). Indeed, at these frequencies the peak variations in the diffraction transition region take place within the test zone. The computation of the maximum radius $R$ of the test zone according to (4), gives the values $R=0.23 \mathrm{~m}$ and $R=0.61 \mathrm{~m}$ at $f=500 \mathrm{mHz}$ and $f=1 \mathrm{GHz}$, respectively. These values should be compared with $R=1 \mathrm{~m}$, the actual test zone radius we consider. A similar analysis for the larger planar arrays give maximum radius way above $1 \mathrm{~m}$. On the other hand, at higher frequencies, i.e., $f=3.5 \mathrm{GHz}$ the error increases considerably for the larger arrays. The maximum interelement distance (5) is $0.115 \mathrm{~m}$ at this frequency which is smaller than the used distance, i.e., $0.126 \mathrm{~m}$. Hence, at higher frequencies, performance is limited by interference caused by the grating lobes.

\section{Conclusion}

An analysis of some of the practical physical limitations of the plane wave synthesis in an anechoic chamber has been presented. The methodology has been on reference measurements in the test zone of a Random Line-Of-Sight Over-The-Air characterization for automotive applications. Two figures of merit where used to define a target accuracy: the relative power at a certain probability of detection level, i.e., $\mathrm{PoD}=0.9$, and the standard deviation of the relative power based on the probability of detection curve. A standard deviation accuracy of the order of $0.5 \mathrm{~dB}$ is feasible in the near-field region over a circular area of radius $R=1 \mathrm{~m}$ at the considered frequency range, i.e., $1.5-3 \mathrm{GHz}$.

\section{ACKNOWLEDGMENT}

This work has been supported by two projects from Sweden's innovation agency VINNOVA, one within the VINN Excellence Center Chase at Chalmers and another via the program Innovative ICT 2013, and by internal support from Chalmers.

\section{REFERENCES}

[1] A. A. Glazunov, V. M. Kolmonen, and T. Laitinen, "MIMO over-theair testing," in LTE-Advanced and Next Generation Wireless Networks: Channel Modelling and Propagation. Hoboken, NJ, USA: Wiley, 2012, pp. 411-441.

[2] P.-S. Kildal, A. A. Glazunov, J. Carlsson, A. Majidzadeh, "Cost-effective measurement setups for testing wireless communication to vehicles in reverberation chambers and anechoic chambers," Antenna Measurements \& Applications (CAMA), 2014 IEEE Conference on , vol., no., pp.1,4, 16-19 Nov. 2014

[3] P.-S. Kildal and K. Rosengren, "Correlation and capacity of MIMO systems and mutual coupling, radiation efficiency and diversity gain of their antennas: Simulations and measurements in reverberation chamber," IEEE Communications Magazine, vol. 42, no. 12, pp. 102-112, Dec. 2004.
[4] P.-S Kildal, "Rethinking the Wireless Channel for OTA testing and Network Optimization by Including User Statistic: RIMP, Pure-LOS, Throughput and Detection Probability," ISAP 2013.

[5] P.H. Lehne, K. Mahmood, A.A. Glazunov, P. Grønsund, P.-S. Kildal,"Measuring user-induced randomness to evaluate smart phone performance in real environments," in Antennas and Propagation (EuCAP), 2015 9th European Conference on , vol., no., pp.1-5, 13-17 April 2015

[6] P.-S. Kildal, A. Hussain, X. Chen, C. Orlenius, A. Skårbratt, J. Asberg, T. Svensson and T. Eriksson, "Threshold Receiver Model for Throughput of Wireless Devices With MIMO and Frequency Diversity Measured in Reverberation Chamber," IEEE AWPL, vol.10, no., pp.1201,1204, 2011.

[7] A. Hussain, P.-S. Kildal, A. A. Glazunov, "Interpreting the Total Isotropic Sensitivity and Diversity Gain of LTE-Enabled Wireless Devices From Over-the-Air Throughput Measurements in Reverberation Chambers," IEEE Access, vol.3, no., pp.131-145, 2015.

[8] P.-S. Kildal, X. Chen, C. Orlenius, M. Franzén, C. Lötbäck Patané, "Characterization of Reverberation Chambers for OTA Measurements of Wireless Devices: Physical Formulations of Channel Matrix and New Uncertainty Formula," IEEE Transactions on Antennas and Propagation, Vol. 60, No. 8, pp. 3875-3891, Aug. 2012

[9] M. Schilliger Kildal, J. Kvarnstrand, J. Carlsson, A.A. Glazunov, A. Majidzadeh and P.-S. Kildal,"Initial Measured OTA Throughput of 4G LTE Communication to Cars with Roof-Mounted Antennas in 2D RandomLOS" (accepted ISAP2015)

[10] M. Schilliger Kildal, A.A. Glazunov, J. Carlsson, J. Kvarnstrand, A. Majidzadeh and P.-S. Kildal, "Measured Probabilities of Detection for 1- and 2 Bitstreams of 2-port Car-roof Antenna in RIMP and Random-LOS", (submitted to EuCAP2016).

[11] A.A. Glazunov, P.-S. Kildal, and M.S. Kildal "Devising a Horizontal Chamber Array for Automotive OTA Tests in Random Line-Of-Sight" (Accepted ISAP2015, Hobart)

[12] D.A Hill, "Theory of near-field phased arrays for electromagnetic susceptibility testing", NBS, Boulder, Colorado, USA, Technical Note 1072, $1984 \mathrm{Feb}$.

[13] J.E. Hansen, Spherical Near-Field Antenna Measurement, IEE Electromagnetic waves series 26, London, U.K.: Peregrinus, 1988.

[14] P.-S. Kildal, X. Chen, M. Gustafsson, S. Zhengzhao, "MIMO Characterization on System Level of 5G Microbase Stations Subject to Randomness in LOS," Access, IEEE , vol.2, no., pp.1064-1077, 2014.

[15] P.-S. Kildal, "Methods and apparatuses for testing wireless communications to vehicles", patent application number EP 14153281.2, 30 januari 2014.

[16] P.-S. Kildal and X. Chen, M. Gustafsson and Z. Shen, "MIMO Characterization on System Level of 5G Microbase Stations Subject to Randomness in LOS," IEEE Access, vol.2, no., pp.1062-1075, 2014.

[17] P.-S. Kildal, C. Orlenius, J. Carlsson, "OTA Testing in Multipath of Antennas and Wireless Devices With MIMO and OFDM," Proceedings of the IEEE, vol.100, no.7, pp.2145-2157, July 2012.

[18] P.-S. Kildal, J.J. Stamnes, "Asymptotic transition region theory for edge diffraction. I. Tracing transition regions via reflectors," in Antennas and Propagation, IEEE Transactions on, vol.38, no.9, pp.1350-1358, Sep 1990.

[19] P-S. Kildal, "Diffraction corrections to the cylindrical wave radiated by a linear array feed of a cylindrical reflector antenna," IEEE Trans. Antennas Propagat., Vol. 32, No. 10, pp. 1111-1116, Oct. 1984.

[20] Per-Simon Kildal, Foundations of Antennas:A Uniïñąed Approach for Line-Of-Sight and Multipath, Kildal Antenn AB, 2015, available at www.kildal.se.

[21] R. Haupt, "Generating a plane wave in the near field with a planar array antenna", Microw. J., pp.152-278, 2003. 